\title{
Cruzando el cuerpo. Dispositivos de frontera y procesos de subjetivación
}

\author{
Crossing the body. Border dispositives \\ and processes of subjectivation
}

PABLO DOMENECH DE LA LASTRA*

\begin{abstract}
Resumen: En este texto buscamos la posibilidad de comprender al cuerpo del migrante como un límite político y ontológico a la construcción de dispositivos fronterizos contemporáneos, centrándonos fundamentalmente en la frontera sur de Europa. Primeramente, daremos cuenta de la emergencia ontológica del migrante como objeto-cuerpo por el poder soberano. Posteriormente, bosquejaremos las formas de aprehensión de este objeto-cuerpo por los poderes gubernamentales, que construyen nuevas formas de sujeción-subjetivación. Finalmente, apuntaremos algunas formas de desbordamiento de los dispositivos fronterizos, para poder hablar de la aparición de un sujeto emancipado, que ejerce de fuerza límite contra las dinámicas globales de vallado. Palabras clave: Soberanía, excepcionalidad, nuda vida, inmunología, tierra de nadie.
\end{abstract}

\begin{abstract}
In this text we look for the possibility to understand the body of migrants as a political and ontological limit to the construction of contemporary border apparatuses, paying a special attention to the European Southern border. First, we take into account the ontological emergence of the migrant as object-body by the sovereign power. Later we sketch the apprehension of this object-body by the governmental powers, building new ways of subjecting-subjectivation. Finally, we point out to some forms of overflow these border apparatuses, so we can speak of the occurrence of an emancipated subject, which exercises the limiting strength against walling global dynamics.
\end{abstract}

Keywords: Sovereignty, exceptionality, nude life, immunology, no man's land.

\section{Introducción}

"We didn't cross the border, the border crossed us". "Nosotros no cruzamos la frontera, la frontera nos cruzó a nosotros", es el eslogan enarbolado desde 2006 por los movimientos

Fecha de recepción: 10/06/2016. Fecha de aceptación: 06/09/2016.

* Doctorando FPU en el Departamento de Filosofía de la Universidad de Murcia pablo.domenech@um.es. Está actualmente investigando las conexiones entre la soberanía, la biopolítica y las migraciones transnacionales, en concreto, las configuraciones de poderes que se despliegan en los dispositivos fronterizos. Ha hecho públicos avances de sus investigaciones en sendos congresos internacionales: «Frontera como espacio de excepción de derecho y violencia inmunológica» en el VI Congreso Internacional e Interdisciplinar de Jóvenes Historiadores. Las Violencias y la Historia, marzo de 2015; y «Deshumanismo en las fracturas ontológicas abiertas por los dispositivos securitarios en las fronteras contemporáneas» en el 8th Conference Beyond Humanism: Posthuman Studies and Technologies of Control, from Nietzsche to trans-, post- and metahumanism, mayo de 2016. 
por los derechos de los inmigrantes asentados en Estados Unidos. Si bien es cierto que esta proposición tiene raíces nacionalistas, recordando la época en que Texas era mexicana, también tiene implicaciones políticas sobre los procesos de subjetivación de los migrantes. Al fin y al cabo, los humanos no somos seres estáticos, sino esencialmente nómadas, que se han expandido por todo el mundo en cuatro grandes olas migratorias. ${ }^{1}$ Las fronteras, en cambio, son entidades estáticas, cuya historia es relativamente corta en lo que se refiere a la humanidad, y devienen de un conjunto de circunstancias políticas contingentes. Cabe preguntarse pues, ¿quién cruza a quién?

En las últimas décadas se ha dado una proliferación de la construcción de dispositivos fronterizos a modo de fortificaciones, muros y vallas. Pero no siempre estuvieron ahí. La lógica política que rige a las fronteras hoy tiene su origen histórico en la paz de Westfalia de 1648, y su origen teórico en el Leviathan de Hobbes de 1651. Entonces las fronteras no eran más que el límite convencional que definía el territorio en el que el Estado soberano podía ejercer su poder de forma absoluta y autónoma, para mantener el orden interno, y con libertad para declarar la guerra a otros Estados soberanos. Si se erigía alguna estructura allí donde lindaba con un territorio ajeno, éstas se orientaban fundamentalmente al control de mercancías. Las aduanas, en otrora dispersas por el territorio, empiezan a concentrarse en la periferia de los Estados, según éstos se van centralizando.

En los primeros siglos del Estado moderno, la inmigración raramente era tenida como una amenaza, sino más bien al contrario, como una fuente de riqueza ${ }^{2}$. En la economía preindustrial la mayor parte de la población estaba atada a la tierra, por lo tanto los dispositivos fronterizos eran inservibles. Por otro lado, a partir del siglo XIX, se dieron lugar migraciones masivas dentro de Europa, por causa de los cambios económicos y demográficos. Sin embargo, ello no implicó la construcción un sistema de control de fronteras ${ }^{3}$. La frontera seguía siendo una mera línea abstracta dibujada por tratados internacionales, que marcaban el alcance de la soberanía territorial.

Con la extensión de los nacionalismos, la frontera adquirirá cierto espesor simbólico. Este registro está marcado por el mito del Estado-nación soberano, "esto es, en la pretensión de establecer un vínculo sagrado entre una determinada etnia y una determinada tierra"4. La frontera adquirirá la cualidad de marcar los límites no tanto de poderes soberanos territorialmente limitados por tratados internacionales, sino comunidades idealmente concebidas alrededor de caracteres lingüísticos, étnicos y raciales. Las nefastas consecuencias de la aplicación del mito de la nacionalidad se pueden observar en el dibujo de las fronteras de Europa central y oriental después de la Primera Guerra Mundial

El gran antecedente histórico de las actuales fronteras fortificadas se encuentra en el Muro de Berlín: un gran dispositivo de seguridad entre regiones que a pesar de pertenecer a la misma nación, se encontraban en las antípodas geopolíticas. Pero su objetivo principal era no militar, sino fundamentalmente el de impedir los movimientos de población entre

1 CAMPILlo MESSEgUER, Antonio (2008): El concepto de lo politico en la sociedad global, Herder, Barcelona, pp. 89-120.

2 SASSEN, Sassen (2013): Inmigrantes y ciudadanos. De las migraciones masivas a la Fortaleza Europa, Siglo XXI, Madrid, pp. 40-43.

3 Ibid., p. 181.

4 CAMPILlO MESSEGUER, Antonio (2008): p. 112. 
las dos regiones enfrentadas. Poco después de la caída del Muro de Berlín, el gran símbolo de la Guerra Fría y de la represión comunista, empiezan a construirse multiplicidad de muros fronterizos para servir la misma función en otras regiones geográficas: limitar el movimiento de personas y poblaciones. La frontera, en esta evolución ha dejado de ser meramente un límite de la soberanía estatal y el territorio nacional, sino que ha adquirido un espesor tanto físico y material, como político y social. Una frontera que en su exuberante materialidad se convierte en un sistema tecnológico de limitación del movimiento de los cuerpos humanos.

\section{Soberanía excepcional}

Lo característico de los nuevos muros fronterizos, es que adolecen de una importante asimetría. Mientras que por un lado dejan pasar libremente capitales, y cómodamente bienes mercantiles, no es así con los seres humanos. Y entre éstos, no ejercen los mismos controles y restricciones a personas adineradas o provenientes de países desarrollados que a personas empobrecidas o de países tercermundistas. Las fronteras fortificadas de este modo se dedican a limitar el movimiento de los cuerpos de ciertas personas, según motivos gubernamentales que en último término intenta justificarse por motivos económicos e ideológicos.

Sin embargo, los dispositivos fronterizos se han demostrado altamente ineficaces, a pesar de su elevado coste, en su objetivo de frenar la inmigración ${ }^{5}$. Las fronteras hoy están sufriendo al mismo tiempo un proceso de fortificación y un proceso de permeabilización. Wendy Brown diagnostica este fenómeno como un síntoma de sobrecompensación psicosocial por parte de soberanías nacionales por demostrar su poder: "los nuevos muros funcionan a menudo como escenificaciones teatrales, proyectando un poder y una eficacia que en realidad no ejercen ni pueden ejercer y que ellos mismos contradicen performativamente." 6 Este fenómeno, tiene como causa el declive de la soberanía ante los nuevos poderes supra y transnacionales en el contexto de la globalización. Entonces, los Estado soberanos, por sobrecompensación, se dedican a expandir su control sobre aquellas instituciones sobre las que todavía mantienen el poder. La frontera es una de ellas, y por eso construyen allí grandes monumentos a la soberanía menguante. Menguante en el contexto de la globalización neoliberal, pero no por ello las soberanías nacionales dejan de ser algunos de los agentes más poderosos del actual panorama global ${ }^{7}$. Y para demostrarlo, levantan las vallas fronterizas como si fueran enormes vallas publicitarias, que más que decirle al extranjero «no eres bienvenido», es un desesperado intento de decirle a los ciudadanos nacionales «el Estado aún te protege» ${ }^{8}$.

El análisis de Brown da razones de peso para la expansión de mecanismos de poder soberano en la frontera. Efectivamente, la fuerza ejercida por el Estado nación en la frontera sigue la lógica de la soberanía. Pero queda incuestionado el mecanismo por el que se despliega el poder soberano, el cual da cuenta de las consecuencias ontopolíticas de la

5 BROWN, Wendy (2015): Estados amurallados, soberanía en declive, Herder, Barcelona, pp. 158-160.

$6 \quad$ Ibid. p. 36.

7 Ibid. p. 30-31.

8 Ibid. p. 135. 
aplicación del poder soberano. En el caso de las fronteras tecnificadas, la soberanía toma la forma de excepcionalidad. Según Carl Schmitt, la soberanía se define por la capacidad de decidir en casos «decisivos», estableciendo los límites entre el estado normal jurídico y el estado de excepción ${ }^{9}$.

De forma abstracta, la lógica westfaliana de la soberanía, permite comprender al espacio exterior contiguo a la frontera nacional como un espacio excepcional. Al fin y al cabo, el estado de derecho sólo es concebible en el interior del territorio. Hacia exterior es donde rige el ius belli, donde reina la excepción pues allí ésta es la excepcionalidad que abre la posibilidad de guerra, que es lo que en último término da sentido a toda unidad política, según $\mathrm{Schmitt}^{10}$.

Si bien la lógica westfaliana permite imaginar una excepcionalidad permanente de la soberanía hacia el afuera de la frontera, el espesor físico que ha adquirido las fronteras tecnificadas contemporáneas ha abierto un verdadero espacio de excepción permanente, donde de facto la violencia soberana es desplegada sin constricciones legales ${ }^{11}$. Con el objetivo de dar una imagen hiperbólica de defensa estatal para reconstituir la imagen moderna de la soberanía nacional, algunos gobiernos abren un espacio excepcional sin precedentes. La hipersecurización de las fronteras ha conllevado la aparición material de una excepcionalidad permanente ${ }^{12}$, la cual permite a los agentes estatales actuar con toda la fuerza que les estaría limitada en el estado de derecho. Esto conlleva, por un lado, el riesgo en la política contemporánea de que la excepcionalidad se generalice en sus formas, y amplíe su campo de acción en detrimento del estado jurídico ordinario ${ }^{13}$. Por otro lado, esta violencia excepcional, produce una serie de marcas sobre el migrante. Se les marca el cuerpo durante los procesos agónicos de cruce, produciendo cicatrices físicas, psíquicas y sociales. Éstas últimas son las que los señalarán racial o culturalmente como extranjeros y sujetos de discriminación.

\section{Emergencia ontológica del cuerpo migrante en la frontera}

El cuerpo migrante emerge en las fronteras contemporáneas como resultado de un proceso de cosificación óntica del mismo. En el proceso de cruce, el migrante es desprendido de sus caracteres subjetuales y es reducido a cuerpo. Este fenómeno ocurre según dos movimientos complementarios: uno que surge del propio migrante, y otro que procede de los mecanismos de rechazo de los Estados nación en las fronteras.

El migrante irregular se desprende en su movimiento transfronterizo de los derechos adquiridos nacionalmente. Mientras que los refugiados usan su nacionalidad como herramienta para migrar, pues les permite pedir asilo, el migrante «económico» debe abandonar formalmente su nacionalidad para evitar las deportaciones, lo que es una decisión nada fácil.

9 SCHMITT, Carl (1998): El concepto de lo político. Texto de 1932 con un prólogo y tres corolarios, Alianza, Madrid, p. 68; AGAMBEN, Giorgio (2004): Estado de excepción. Homo sacer II, 1, Pre-Textos, Valencia, pp. 54-55.

10 SCHMITT, Carl (1998): pp. 62-65.

11 El mejor ejemplo de este espacio de excepción permanente es la tierra de nadie que se abre entre las vallas exteriores de Ceuta y Melilla, donde, a pesar de ser territorio soberano español, actúan sin restricciones tanto policías marroquíes como españoles.

12 DE LUCAS, Javier (2015): Mediterráneo: el naufragio de Europa, Tirant Humanidades, Valencia, p. 19

13 Ibid. pp. 36-37; AGAMBEN, Giorgio (2004): pp. 25, 32. 
El camino del migrante irregular, desde su lugar de origen, hasta su destino, esquivando los dispositivos fronterizos, es de gran peligro, tal y como relatan las crónicas de fallecidos y desaparecidos ${ }^{14}$. La búsqueda de unas mejores condiciones de vida entraña un peligro nada desdeñable de muerte. Los migrantes ponen su vida en riesgo, con su cuerpo como única potencia. En su proceso de cruce, dejan de lado sus derechos nacionales.

Por otro lado, los países de destino de las migraciones imponen una serie de condiciones que desnudan a los migrantes irregulares de aquellos derechos que les quedan. Primeramente los Estados ricos imponen una estratificación de los extranjeros. La distinción más notoria es la que se impone entre los extranjeros documentados y aquellos que, por su estatus racial y económico, no se les permite esta vía cómoda de migración. Una vez establecido un grupo migratorio carente de derechos de migrar, se les impone, a aquellos que lo consiguen, una serie de condiciones que los despojan de los derechos más básicos. Además de las fronteras fortificadas, podemos destacar prácticas policiales de acoso, detenciones, deportaciones y encierros ${ }^{15}$; todas ellas dirigidas a establecer el carácter de sujeto sin derechos en el migrante marcado en la frontera. En un doble movimiento, los migrantes clandestinos se convierten en apátridas.

Las nuevas fronteras fortificadas se utilizan para despojar de los últimos vestigios de derechos a los migrantes indeseados. Los dispositivos fronterizos son una maquinaria de producción de apátridas. Ponen en marcha los poderes soberanos del Estado para desnudar a los migrantes de su dignidad y sus derechos más básicos, dejando la abstracta desnudez de ser humano ${ }^{16}$. Hoy podemos decir de nuevo, con los millones de migrantes irregulares y refugiados que hay en el mundo, que el apátrida se ha convertido en una figura central de la política contemporánea. Se trata de personas que, sin poder apelar a los Derechos Humanos, pues carecen de un Estado que los defienda por ellos, se les desprende del más básico de los derechos: el derecho a tener derechos. Este vacío jurídico al que se somete apátrida se da como consecuencia de la estricta compartimentación y saturación del espacio en unidades políticas autónomas.

Es precisamente en las fronteras tecnificadas donde la violencia institucional hacia el apátrida aparece más intensa. El dispositivo fronterizo contemporáneo crea precisamente un espacio de excepción permanente como mecanismo de legitimación para la violencia soberana que deshumaniza a los migrantes. Esto produce que se «desnude» de todo derecho a las personas que intentan cruzar. No se los reconoce como sujetos de derecho sino enemigos de excepción, y por tanto se puede ejercer contra ellos una fuerza propia del estado de excepción. Los migrantes se convierten en la nuda vida que Giorgio Agamben ponía en el núcleo de su teoría del homo sacer. Nuda vida es el cuerpo desprovisto de todo derecho, ex-puesto a la violencia del bando soberano. "La relación política originaria es el bando (el estado de

14 Sirva de ejemplo el informe: INTERNATIONAL ORGANIZATION FOR MIGRATION (IOM), BRIAN, Tara y LACKZO, Frank (eds.) (2014): Fatal Journeys. Tracking Lives Lost during Migration, Ginebra, pp. 20-21. Disponible en: http://publications.iom.int/bookstore/free/FatalJourneys_CountingtheUncounted.pdf [consultado el 14 de abril de 2016]

15 CAMPAÑA ESTATAL POR EL CIERRA DE LOS CIE (2014): Paremos los vuelos. Las deportaciones de inmigrantes y el boicot a Air Europa, Cambalache, Oviedo, pp. 23-42.

16 ARENDT, Hannah (2004): The origins of totalitarism, Schocken Books, New York, pp. 377-378. 
excepción como zona de indistinción entre exterior e interior, exclusión e inclusión)" ${ }^{17}$. Y el apátrida frente a la frontera se ha convertido hoy en el habitante por antonomasia de la excepcionalidad.

Así, el migrante enfrentado a los dispositivos fronterizos, no es sólo apátrida, sin territorio donde pueda tener derechos, sino también es nuda vida sobre la que la soberanía despliega su poder y justifica su legitimidad excepcional. Nuda vida es el inmigrante que es deportado sumariamente, sin mediar su derecho de asilo. Nuda vida es aquél encerrado en un Centro de Internamiento de Extranjeros (CIE), que reproduce la lógica excepcional del campo de concentración ${ }^{18}$. Nuda vida es el ahogado en el mediterráneo del no se molesta en rescatar.

La aparición del migrante como nuda vida es la aparición óntico-ontológica del cuerpo del mismo. El migrante irregular, enfrentado al poder excepcional, se ex-pone como cuerpo. Se ex-pone a la violencia soberana. Se ex-pone a la posibilidad de la muerte. Su cuerpo es el objeto contra el que se levantan los muros. Es políticamente cuerpo y nada más. No es ciudadano ni de dentro ni de fuera. Carece de todos los derechos nacionales y, en el límite, en la frontera material, de los internacionales.

Pero la frontera no sólo abre un espacio de excepción donde el migrante se reduce a mero cuerpo expuesto a la violencia soberana. También abre la posibilidad de resistencia y límite de las acciones excepcionales. Ante la debilidad del estado de derecho, el cuerpo aparece como campo de batalla entre las fuerzas de movimiento y las fuerzas de rechazo. Pues el migrante es plenamente nuda vida sólo cuando está sujeto por los mecanismos de control soberano: durante las devoluciones en caliente o en los CIE. Allí, sólo tiene su cuerpo como fuerza defensiva. Y tal vez sea allí donde el cuerpo del inmigrante pueda convertirse en el fundamento ontológico de las luchas contra las medidas represivas del Estado excepcional. Pero cuando está fuera de las instituciones excepcionales, los migrantes cuentan con toda una serie de mecanismos de organización y cuidado, que les permite construir sus proyectos vitales y desarrollar una subjetividad individual y colectiva que circunnavega o se enfrenta a los poderes coactivos del Estado.

\section{Resemantización biopolítica del cuerpo inmigrante}

A pesar de que las tecnologías fronterizas acaben en un momento dado reduciendo al migrante a mero cuerpo viviente, no por ello actúan ciegamente. Las fronteras son mecanismos precisos de selección de sujetos. La violencia excepcional en el muro no es ejercida de igual manera a todos los sujetos que se enfrentan a ella. Los dispositivos fronterizos son mecanismos al mismo tiempo tanto de inclusión como de exclusión, en tanto que seleccionan y filtran el modo de cruce de cada sujeto, la intensión de la fuerza soberana y la clasificación selectiva de los migrantes ${ }^{19}$. La selección se realiza según dos criterios fundamentales: el mercado de trabajo y el deseo de homogeneidad de la comunidad.

17 AGAMBEN, Giorgio (1998): Homo sacer. El poder soberano y la nuda vida, Pre-Textos, Valencia, p. 230.

18 MEZZADRA, Sandro y NEILSON, Brett (2013): Border as Method, or, the Multiplication of Labor, Duke University Press, Durham and London, pp. 133-135.

19 Ibid.p. 7. 
Si bien las fronteras han cambiado poco en los últimos años en lo que se refiere a la circulación de los bienes del capitalismo global, hay uno de ellos que es inherente al cuerpo humano: el trabajo vivo, la mano de obra. El trabajo viviente es una commodity inseparable de los cuerpos vivos, y por ello el capital transnacional construye dispositivos de control con el objetivo de explotarlos ${ }^{20}$. Es precisamente contra los cuerpos vivos contra lo que están construidas las hiperbólicas fronteras materiales, no contra capitales ni bienes de consumo. $\mathrm{Y}$ es en la apertura de una excepcionalidad fronteriza permanente precisamente donde los migrantes son reducidos a meros cuerpos vivientes.

Las necesidades económicas del mercado capitalista global lleva a los Estados contemporáneos, ante la exigencia de mantenerse competitivos, a crear diferentes clases sociales y de trabajadores dentro de su territorio. Como en los países del Primer Mundo la explotación laboral de los ciudadanos de derecho daría lugar a un gran rechazo social, se ha implementado un régimen de semiesclavitud estructural a los migrantes del Tercer Mundo. Las legislaciones de extranjería y las prácticas policiales de identificación, detención y deportación, dan lugar a una estratificación de los habitantes del territorio en función de su grado de naturalización ${ }^{21}$. "En este sentido, la nacionalidad funciona también como una frontera que marca los límites de la membresía y excluye a los otros, aquellos con los que no se tiene obligaciones."22 En el extremo opuesto a los ciudadanos nacionales, tomados como sujetos de derecho, los migrantes «ilegales» o «clandestinos» son un nuevo lumpenproletariat del siglo XXI:

Se configura así un estatus jurídico segregador que determina tener que vivir de manera permanente en una posición social subalterna, con todas las implicaciones negativas que esta situación entraña en términos de oportunidades de bienestar. [...] Este tipo de funcionalidad sería extensible también a los sistemas de control de fronteras, que no persiguen tanto ordenar las migraciones de la gente como optimizar los mecanismos de explotación de los trabajadores transnacionales ${ }^{23}$.

Las fronteras en este sentido fabrica una masa de trabajadores disponibles a para los puestos de trabajo más serviciales. El mercado laboral global se nutre de la migración masiva de mano de obra barata desde países pobres. La gran inflación de fronteras exteriores e interiores, y una heterogeneización de los espacios y los tiempos laborales y cívicos, es impuesta por el mercado global. Las fronteras fortificadas no son un mero mecanismo de exclusión, sino que su modus operandi principal es el de la inclusión diferencial ${ }^{24}$. Las fronteras son nuevos mecanismos de selección de personal para las economías globalizadas y crean nuevas clases sociales en el seno de los Estados democráticos.

20 Ibid. p. 19.

21 DE LUCAS, Javier (2015): pp. 30-32.

22 VELASCO, Juan Carlos (2015): «La justicia y los derechos en un mundo globalizado», en: ARCOS, Federico (ed.), La justicia y los derechos en un mundo globalizado, Dykinson, Madrid, pp. 49-74, p. 51.

23 Ibid. p. 53.

24 MEZZADRA, Sandro y NEILSON, Brett (2013): pp. 157-166. 
El segundo criterio de la selectividad de las fronteras contemporáneas es el proyecto de preservar una mitológica homogeneidad nacional. La principal función de este criterio es la legitimación ideológica y política de los costosos muros fronterizos. Si bien la regulación del mercado laboral pueda cumplir una función de justificación de medidas protectoras, será la búsqueda de comunidades ideales lo que legitima ideológicamente los muros fronterizos. La identidad de las naciones modernas es una construcción simbólica y cultural que es atravesada por categorías raciales. El pensamiento político occidental, hoy mundialmente hegemónico, que pivota alrededor de la figura del Estado nación, hunde sus raíces ideológicas en el mito de la coincidencia entre el territorio y la raza, etnia o cultura ${ }^{25}$. Esta mitología nacionalista, se despliega hoy en las fronteras como un conjunto de fantasías: la fantasía de que la unidad nacional es algo evidente, que es pura e inocente; que su única forma de mantenerse así es conteniendo a los extranjeros que, por definición, son peligrosos; y que, efectivamente, se puede mantener aislada ${ }^{26}$.

Por estas razones la alteridad que representan los extranjeros no puede ser siempre neutralizada a través de la asimilación. Las naciones se ven enfrentadas a la necesidad de preservar una comunidad interna a través mecanismos de inmunidad, tal y como nos explica Roberto Esposito ${ }^{27}$. En este sentido, los extranjeros adquieren los caracteres biopolíticos de exógenos, e incluso patógenos, desde las ideologías nacionalistas. Ante las migraciones masivas que produce la globalización, los Estados se enfrentan a decidir entre la asimilación o la expulsión de los migrantes -la tercera vía, la de la exterminación, ya ha sido puesta en práctica en el pasado de forma nefasta. El cuerpo del Estado, el Leviathan compuesto por los cuerpos de los ciudadanos que nos dibujaba Hobbes en 1651, busca preservar su armonía biótica, sea de caracteres raciales, étnicos o culturales. Pero para conseguirla, tiene que someterse a agresivos tratamientos. Uno de estos tratamientos protectores es el de desplegar dispositivos profilácticos allí donde hay contacto con comunidades exteriores. Los muros fronterizos son los más obvios objetos preventivos.

Los mecanismos de control de las poblaciones extranjeras en forma de biopolítica nacionalista, es un síntoma más de la relevancia del cuerpo vivo del migrante. Por un lado, los Estados se enfrentan a las necesidades del mercado global, en el que cada vez pueden controlar menos. Y por otro lado pretende fundamentar su legitimidad en el ideal mitológico de una unidad nacional, cada vez más difícil de justificar en términos bióticos. Desde estos dos frentes, se fabrican mecanismos de resemantización biopolítica de los cuerpos migrantes. Más allá de nuda vida, son trabajadores explotables, y agentes exógenos que legitiman las estructuras biopolíticas del Estado.

Pero, a la inversa, este biopolítico «dar-de-nuevo-sentido» al cuerpo ex-puesto del migrante es en parte causa de la apertura ontológica del cuerpo humano por medio de la soberanía excepcional. Las necesidades inmunológicas de las naciones en decadencia identitaria exigen una refundación permanente que conducen a medidas heterogéneas de exclusión de ciertas poblaciones étnicamente marcadas. Por otro lado, los dispositivos fronterizos son mecanismos promovidos por el mismo mercado laboral global. La biopolítica de las fronteras contemporáneas provee de sentido a los cuerpos desnudos de derechos, después

25 CAMPILLO MESEGUER, Antonio (2008): 103-106.

26 BROWN, Wendy (2015): 166-179.

27 ESPOSITO, Roberto (2009): Comunidad, inmunidad, biopolitica, Herder Editorial, Barcelona, pp. 81-93. 
de habérselos sustraído, con el objetivo de controlarlos, según las necesidades políticas o económicas. Y ambos criterios gubernamentales que justifican la tecnificación fronteriza -el económico y el inmunológico-, usan como herramienta gestora la marca-cicatriz biopolítica que la soberanía excepcional impone sobre los cuerpos migrantes en el cruce de fronteras. Pero al igual que la emergencia del cuerpo desnudo en la excepción fronteriza preconizaba una forma homóloga de resistencia, la biopolítica de migraciones permite nueva formas de empoderamiento por parte de los colectivos que cruzan las fronteras.

\section{El cuerpo como límite de la frontera}

Hemos querido demostrar que el cuerpo migrante es el objeto central de las políticas que se despliegan en las fronteras fortificadas contemporáneas. Por un lado aparece fugazmente como nuda vida por la violencia de una soberanía que se despliega en la frontera como fuerza excepcional. Por otro lado, el control biopolítico de las poblaciones extranjeras carga de ciertos atributos vitales de los cuerpos migrantes. Pero en ningún caso podemos considerar estos dos momentos como aislados lógica o políticamente. Así como tampoco podemos asumir que estas dos instancias de poder trabajan complementariamente y en armonía.

Aun así, en ningún momento el migrante es un agente pasivo. Es su cuerpo contra el que se opone la frontera. Pero es su cuerpo el que la atraviesa y cruza. Las políticas de fronteras viene siempre acompañada por políticas de cruce: técnicas y estrategias colectivas diseñadas para sortear las mutables reglas de la frontera. Los migrantes, dentro de sus capacidades individuales y colectivas, diseñan toda una serie de prácticas con el objetivo de una vida mejor en una nación que no los quiere acoger. Estas prácticas, al mismo tiempo, no están desprovistas de violencia. Violencia contra las fronteras, y violencia interna en las comunidades de migrantes.

El cuerpo de los migrantes se ve hendido y herido por los dispositivos represivos desplegados en las fronteras. Son tratados como parásitos desde las instancias inmunológicas del Estado. Se les entrena y selecciona para mayor provecho de un mercado laboral necesitado de mano de obra barata, endurecida y dócil. Se les desnuda de todo derecho, se les convierte en nuda vida, cuando son deportados sumariamente, arrojados de nuevo al otro lado de la valla, sin atender los Derechos Humanos básicos que los países del Primer Mundo prometen proteger. Entonces, los cuerpos migrantes, se convierten en una doble herida. Primero, la frontera deja cicatrices en los inmigrantes; cicatrices físicas, psicológicas y simbólicas, que afectan sus canales de subjetivación individual y colectiva. Segundo, la presencia de estos hombres y mujeres marcados también dibujan una herida en el tejido social. De este modo, la violencia contra los migrantes se internaliza en la nación que se pretende protege. Las fronteras, a pesar de su sistemática porosidad, acaban afectando la propia movilidad de los ciudadanos nacionales ${ }^{28}$. Proliferan los muros interiores, haciendo problemática una distinción clara entre exterior e interior ${ }^{29}$. La creación de mecanismos de monitorización del movimiento de los sujetos y de estratificación social en función

28 DE LUCAS, Javier (2015): p. 53.

29 MEZZADRA, Sandro y NEILSON, Brett (2013): p. 7. 
de su grado de «extranjería», está constriñendo también los derechos de los ciudadanos nacionales: "desde la perspectiva de la ciudadanía, tratar a los inmigrantes como a seres «ilegales» acaba devaluando a la ciudadanía misma"30.

El cuerpo del migrante es el vórtice de esta doble herida, la herida en el sujeto y la herida en la sociedad. Pero puede ser también la piedra angular de nuevas formas de subjetividad política y ontológica. Las fronteras abren un espacio de negatividad ontológica en los lugares de excepcionalidad permanentes. La tierra de nadie que abren las fronteras contemporáneas son medios de proliferación de lo inmundo, fragmentos de no-mundo, atravesados por cierta pulsión de muerte ${ }^{31}$, si entendemos, con Jean-Luc Nancy, que el mundo es una totalidad de sentido ${ }^{32}$. En este sentido, la creación de mundo ex nihilo, como lugar que habitar y dotar de sentido, es el reverso la apertura de un espacio de nadie, que la excepcionalidad fronteriza devuelve al nihil. Ahí, en esa nada que es la tierra de nadie, emerge el cuerpo migrante como objeto de violencia deshumanizadora propia de la excepción soberana, y como sujeto de biopolíticas de gestión del mercado laboral y de inmunización de identidades nacionales.

Así, la maquinaria de las fronteras impone límites a la justicia, pero permite al mismo tiempo la administración de la justicia, y al ser tanto un dispositivo de inclusión como de exclusión, permite la modulación de diferentes formas de subjetividad ${ }^{33}$. Pero, tal y como hemos querido demostrar, es crucial la importancia que adquiere el cuerpo migrante en la formación de mecanismos de subjetivación individual y colectiva en las políticas de cruce, en tanto que es el objeto-sujeto de las políticas de frontera.

El cuerpo es destacado ónticamente en la frontera. Y en este proceso, el cuerpo migrante emerge como nuda vida en la ontología negativa de la excepción fronteriza. Pero como emerge precisamente como cuerpo y sólo como cuerpo, es el principio de toda una nueva ontología que construya sus propios códigos de subjetivación más allá de la violencia excepcional y el control biopolítico. El cuerpo es una apertura ontológica de un espacio de sentido, "el cuerpo da lugar a la existencia." Existencia más allá o más acá de toda esencia. "Por eso es que la ontología del cuerpo es la ontología misma: ahí el ser no es nada previo o subyacente al fenómeno. El cuerpo es el ser de la existencia." ${ }^{34}$ Sin llegar a compartir plenamente las implicaciones heideggerianas, si compartimos con Nancy la necesidad de tener en cuenta la existencia corpórea de los sujetos en toda construcción ontopolítica, sobre todo en el caso de las políticas fronterizas. Si bien son sus técnicas de control y violencia las que hace emerger el cuerpo migrante como lugar de una ontología negativa, de sustracción de sentido y fuerzas, por otro lado, toda emergencia del cuerpo es la posibilidad de dar lugar a una reconstitución ontológica de la existencia.

Por tanto, el cuerpo se constituye como el límite último de la frontera. La frontera no deja de estrellarse contra el único elemento que no puede atravesar, el cuerpo vivo de los sujetos en movimiento. La frontera puede retenerlos, dañarlos y, en el límite, asesinarlos. Pero, ¿puede por ello atravesar todo su espesor ontológico? Es lo que aquí hemos puesto en

30 SASSEN, Sassen (2013): p. 13.

31 NANCY, Jean-Luc (2003): La creación del mundo o la mudialización, Paidós, Barcelona, p. 16.

32 Ibid., p. 29.

33 MEZZADRA, Sandro y NEILSON, Brett (2013): p. 269.

34 NANCY, Jean-Luc (2003): Corpus, Arena Libros, Madrid,p. 16. 
duda. Y queremos con ello dar lugar al análisis sobre la confluencia en el cuerpo migrante de las distintas fuerzas que se ponen en tensión desde la materialidad de su movimiento a través y contra las fronteras, y a partir de ello, abrir la posibilidad de reescribir los códigos de subjetivación que se da cuando los migrantes cruzan la frontera o, como dicen los migrantes mexicanos en Estados Unidos, como la frontera cruza los migrantes.

\section{Bibliografía}

Agamben, Giorgio (1998): Homo sacer. El poder soberano y la nuda vida, Pre-Textos, Valencia.

Agamben, Giorgio (2004): Estado de excepción. Homo sacer II, 1, Pre-Textos, Valencia.

Arendt, Hanna (2004): The origins of totalitarism, Schocken Books, New York.

Brown, Wendy (2015): Estados amurallados, soberanía en declive, Herder, Barcelona.

Campaña Estatal por el Cierra de los CIE (2014): Paremos los vuelos. Las deportaciones de inmigrantes y el boicot a Air Europa, Cambalache, Oviedo.

Campillo Meseguer, Antonio (2008): El concepto de lo político en la sociedad global, Herder, Barcelona.

De Lucas, Javier (2015): Mediterráneo: el naufragio de Europa, Tirant Humanidades, Valencia. Esposito, Roberto (2009): Comunidad, inmunidad, biopolítica, Herder Editorial, Barcelona. International Organization for Migration (IOM), Brian, Tara y Lackzo, Frank (eds.) (2014): Fatal Journeys. Tracking Lives Lost during Migration, Ginebra.

Mezzadra, Sandro y NEILSON, Brett (2013): Border as Method, or, the Multiplication of Labor, Duke University Press, Durham and London.

Nancy, Jean-Luc (2003): Corpus, Arena Libros, Madrid.

Nancy, Jean-Luc (2003): La creación del mundo o la mudialización, Paidós, Barcelona.

Sassen, Sassen (2013): Inmigrantes y ciudadanos. De las migraciones masivas a la Fortaleza Europa, Siglo XXI, Madrid.

Schmitt, Carl (1998): El concepto de lo político. Texto de 1932 con un prólogo y tres corolarios, Alianza, Madrid.

Velasco, Juan Carlos (2015): «La justicia y los derechos en un mundo globalizado», en: Arcos, Federico (ed.), La justicia y los derechos en un mundo globalizado, Dykinson, Madrid. 
